\title{
Ação de extratos de plantas na oviposição e na mortalidade da traça-das-crucíferas
}

\author{
Action of plants extracts on oviposition and on mortality of diamondback moth \\ Sônia Thereza Bastos Dequech ${ }^{\mathrm{I}}$ Rafael Egewarth ${ }^{\mathrm{II}}$ Carla Daniele Sausen ${ }^{\mathrm{II}}$ \\ Vinícius Soares SturzaII Leandro do Prado Ribeiro ${ }^{\mathrm{II}}$
}

\begin{abstract}
A traça-das-crucíferas, Plutella xylostella (L.) (Lepidoptera: Yponomeutidae), é uma das principais espécies de inseto-praga de plantas da família Brassicaceae no Brasil e no mundo. A utilização de extratos de plantas inseticidas no controle desse inseto é uma alternativa promissora. O presente trabalho teve como objetivo avaliar, em laboratório, o efeito de extratos de plantas na oviposição e na mortalidade de $\boldsymbol{P}$. xylostella. Para análise do efeito sobre a oviposição, pedaços de folhas de couve foram expostos, em gaiola, aos seguintes tratamentos: extratos aquosos $(10 \% \mathrm{p} / \mathrm{v})$ de folha de cinamomo (Melia azedarach), de ramo de cinamomo, de pó-de-fumo (Nicotiana tabacum), além de DalNeem (produto comercial à base de Azadirachta indica), a 10\% v/v. No interior da gaiola, foram liberados 50 adultos de $\boldsymbol{P}$. xylostella e, após 48h, foi contado o número de ovos por folha, em sete repetições. Um segundo ensaio constou da exposição de três larvas aos extratos referidos anteriormente em tubos de vidro com um pedaço de folha de couve previamente tratado. Foram utilizados três tubos por tratamento, com quatro repetições. O número de larvas mortas foi avaliado diariamente. Os extratos aquosos de folha de cinamomo, de ramo de cinamomo e de pó-de-fumo, além de DalNeem, reduzem a oviposição de P. xylostella e a utilização de extrato de pó-de-fumo, de DalNeem e de ramo de cinamomo resultam em controle eficiente de larvas de $\boldsymbol{P}$. xylostella a partir do segundo, do quinto e do sexto dias após a aplicação, respectivamente.
\end{abstract}

- NOTA -

\begin{abstract}
The diamondback moth, Plutella xylostella (L.) (Lepidoptera, Yponomeutidae), is one of the most important insect pest species of the Brassicaceae family plants in Brazil and in the world. The use of insecticidal plants extracts in the control of this insect is a promising alternative. Therefore, this research goal is to evaluate, in laboratory, the plants extracts effect on the oviposition and mortality of $\boldsymbol{P}$. xylostella. In order to analyze the effect on the oviposition, kale leaf pieces were treated, in cage, as following: aqueous extracts $(10 \% \mathrm{p} / \mathrm{v})$ of leaf of chinaberry (Melia azedarach), of branch of chinaberry, of tobacco powder (Nicotiana tabacum), besides DalNeem (commercial product, based on Azadirachta indica), at 10\% $v / v$. Inside the cage, 50 adults of $\boldsymbol{P}$. xylostella were put and, $48 \mathrm{~h}$ later, the egg number per leaf was counted, in seven repetitions. A second assay was consisted of the exposition of three larvae to the same cited extracts, in glass tube with a kale leaf piece previously treated. Three glass tubes were used per treatment, with four repetitions. The number of death larvae was evaluated daily. The aqueous extracts of chinaberry leaf, chinaberry branch and tobacco powder, besides DalNeem, reduce the oviposition of $\boldsymbol{P}$. xylostella and the use of tobacco powder extract, DalNeem and chinaberry branch extract result in efficient control of $\boldsymbol{P}$. xylostella larvae after the second, fifth and sixth days after application, respectively.
\end{abstract}

\section{Palavras-chave: Plutella xylostella (Lepidoptera: Yponomeutidae), Melia azedarach, Azadirachta indica.}

Key words: Plutella xylostella (Lepidoptera: Yponomeutidae), Melia azedarach, Azadirachta indica.

A traça-das-crucíferas, Plutella xylostella (Linnaeus, 1758) (Lepidoptera, Yponomeutidae), é

IDepartamento de Defesa Fitossanitária, Universidade Federal de Santa Maria (UFSM), 97105-900, Santa Maria, RS, Brasil. Email: soniabd@terra.com.br. Autor para correspondência.

${ }^{\text {IIC } C u r s o ~ d e ~ A g r o n o m i a, ~ U F S M, ~ S a n t a ~ M a r i a, ~ R S, ~ B r a s i l . ~}$ 
considerada, na Ásia e nas Américas, uma praga que causa sérios danos aos cultivos de brássicas, sendo a principal praga do repolho (CASTELO BRANCO \& FRANÇA, 2001; MACHADO et al., 2007). O uso de inseticidas tem sido a principal medida de controle de P. xylostella (CASTELO BRANCO \& FRANÇA, 2001). Uma das alternativas para a busca da substituição de inseticidas sintéticos por inseticidas naturais é o uso de substâncias secundárias (aleloquímicos) presentes em plantas com atividade inseticida. Os derivados botânicos podem causar diversos efeitos sobre os insetos, tais como repelência, inibição de oviposição e da alimentação, alterações no sistema hormonal, causando distúrbios no desenvolvimento, deformações, infertilidade e mortalidade nas diversas fases (ROEL, 2001).

A família Meliaceae destaca-se entre as mais utilizadas como fonte de aleloquímicos, sendo que o nim (Azadirachta indica A. Juss), planta com atividade inseticida mais estudada no mundo, pertence a essa família. Entre os efeitos causados aos insetos pelo ingrediente ativo mais potente do nim, a azadiractina, incluem a inibição (ou deterrência) da alimentação e de oviposição (MARTINEZ, 2002). Ainda entre as meliáceas, o cinamomo (Melia azedarach $\mathrm{L}$.) vem sendo avaliado por apresentar compostos limonóides igualmente presentes no nim, como a azadiractina (VIEIRA\& FERNANDES, 1999). LOVATTO et al. (2004) referem-se à utilização de solanáceas como controladoras de insetos, por apresentarem metabólitos secundários com significativa toxicidade. Entre estas, o fumo, Nicotiana tabacum L., que possui os alcalóides nicotina, nornicotina e anabasina (VIEIRA \& FERNANDES, 1999).

O presente trabalho foi realizado com o objetivo de avaliar o efeito de extratos de plantas com atividade inseticida na mortalidade e na oviposição de P. xylostella, em condições de laboratório. Os experimentos foram realizados no Laboratório de Entomologia, do Departamento de Defesa Fitossanitária, do Centro de Ciências Rurais, da Universidade Federal de Santa Maria (UFSM), e constaram das seguintes etapas:

\section{Criação da traça-das-crucíferas}

Para o início da criação, foram coletadas posturas e larvas de $\boldsymbol{P}$. xylostella de plantas de couveflor (Brassica oleracea L. var. botrytis), que foram mantidas em gaiolas medindo $0,47 \mathrm{~m}$ de comprimento $\mathrm{x}$ 0,31m de largura x 0,34m de altura para obtenção dos adultos. Os adultos foram transferidos para gaiolas plásticas $(0,47 \mathrm{~m} \times 0,31 \mathrm{~m} \times 0,34 \mathrm{~m})$ a fim de serem obtidas larvas, com idade conhecida, para utilização nos experimentos. Os adultos foram alimentados com solução de mel e água (10\%) e as larvas foram alimentadas com folhas de couve (Brassica oleracea var. acephala).

Extratos de plantas inseticidas

Foram utilizados extratos aquosos de folha de cinamomo, de ramo de cinamomo e de pó-de-fumo. As estruturas vegetais de cinamomo foram coletadas no campus da UFSM, em outubro de 2006. O pó-defumo foi obtido junto às indústrias fumageiras da região, sendo constituído de resíduos do processamento de folhas de fumo, após a moagem das mesmas. Para preparo dos extratos, na concentração de $10 \%$ (peso/ volume), seguiu-se a metodologia proposta por VENDRAMIM \& CASTIGLIONI (2000). Outro tratamento utilizado nos experimentos, na concentração de $10 \%$ v/v, foi o produto comercial DalNeem, à base de frutos maduros de A. indica, com aproximadamente 3.000ppm de princípio ativo e sob a forma de óleo emulsionável. Em todos os ensaios foi utilizado um tratamento à base de água como testemunha.

Atividade dos extratos sobre a oviposição

Foi realizado um experimento no qual foi avaliada a atividade dos extratos na oviposição de $\boldsymbol{P}$. xylostella. Para tanto, foi utilizada uma gaiola plástica $(0,32 \mathrm{~m} \times 0,20 \mathrm{~m} \times 0,34 \mathrm{~m})$, com duas janelas teladas, na qual foram dispostos, ao acaso, cinco pedaços com área de $36 \mathrm{~cm}^{2}(6 \mathrm{~cm} \times 6 \mathrm{~cm})$ de folhas de couve previamente tratados com os extratos aquosos de folha de cinamomo, de ramo de cinamomo, de pó-de-fumo, além de DalNeem e de água. No interior da gaiola, foram liberados 50 adultos de $\boldsymbol{P}$. xylostella. A gaiola foi mantida em câmara incubadora, onde os insetos foram mantidos à temperatura de $25 \pm 1^{\circ} \mathrm{C}$, umidade relativa de $70 \pm 10 \%$ e fotofase de 14 horas. Após $48 \mathrm{~h}$, foi contado, com auxílio de microscópio estereoscópico, o número de ovos em cada folha.

\section{Efeito dos extratos na mortalidade das larvas} Pedaços de folhas de couve de $12 \mathrm{~cm}^{2}$ (3cm $\mathrm{x} 4 \mathrm{~cm}$ ) foram imersos nos mesmos tratamentos mencionados no ensaio anterior, por 20 segundos, e posteriormente foram distribuídos sobre papel toalha e mantidos em condição ambiente para evaporação do excesso de líquido. Três larvas recém-eclodidas, de até $24 \mathrm{~h}$, foram colocadas em tubos de vidro $(2,5 \mathrm{~cm}$ de diâmetro x 8,5cm de altura), com um pedaço de folha de couve previamente tratado. Os tubos foram fechados com algodão umedecido, para evitar a saída das larvas, e levados à câmara incubadora, em condições já mencionadas anteriormente. Foram realizadas 
observações diárias com o objetivo de avaliar o efeito dos tratamentos sobre a mortalidade das larvas. As folhas foram substituídas por outras não-tratadas apenas quando estavam ressecadas.

O delineamento experimental utilizado foi o inteiramente casualizado com cinco tratamentos. Foram realizadas sete repetições no primeiro ensaio e quatro repetições no segundo. Os resultados obtidos foram submetidos à análise de variância, com as médias comparadas pelo teste de Tukey ao nível de significância de $5 \%$. No segundo ensaio, os dados de mortalidade (\%) dos tratamentos e da testemunha foram utilizados para o cálculo da eficiência dos inseticidas por meio da fórmula de Abbott (ABBOTT, 1925).

Todos os extratos avaliados (folha de cinamomo, ramo de cinamomo, pó-de-fumo e DalNeem) reduziram a oviposição de $\boldsymbol{P}$. xylostella em folhas de couve $\left(F_{(4,24)}=5,55 ; P=0,0026\right)$ (Figura 1). Resultado semelhante foi obtido por CHEN et al. (1996), os quais reportaram que o número de ovos de $\boldsymbol{P}$. xylostella foi menor em plântulas de colza (Brassica napus L.) tratadas com extratos de frutos M. azedarach, em comparação à testemunha. Além disso, MEDEIROS et al. (2005) obtiveram altos valores de porcentagem média de deterrência na oviposição de $\boldsymbol{P}$. xylostella para extratos aquosos a $10 \%$ p/v de folhas de $N$. tabacum (99,5\%) e de $\boldsymbol{A}$. indica (89,1\%). Da mesma forma, porém testando extratos em concentrações mais baixas, TORRES et al. (2006) obtiveram porcentagem média de repelência de $10,4 \%$, testando extratos aquosos de amêndoas de $\boldsymbol{A}$. indica e $16,0 \%$ de frutos de $\boldsymbol{M}$. azedarach. Por outro lado, LIANG et al. (2003) observaram que três inseticidas comerciais à base de nim (Agroneem, Ecozin e Neemix) não apresentaram ação como deterrentes na oviposição de $\boldsymbol{P}$. xylostella.

As larvas expostas a extratos de pó-de-fumo apresentaram um alto índice de mortalidade já a partir do primeiro dia, o que demonstra o efeito imediado dos metabólitos presentes em plantas de fumo. Por outro lado, o DalNeem não causa a morte do inseto imediatamente, dado o seu efeito fisiológico, afetando a ecdise e reduzindo o consumo de alimento (MARTINEZ, 2002). Do primeiro ao sexto dia após a aplicação dos tratamentos ocorreu um aumento gradual da eficiência de controle das larvas expostas aos extratos de ramo e à folha de cinamomo (Tabela 1).

A partir do segundo dia após a aplicação dos tratamentos não houve diferença significativa na eficiência de controle das larvas expostas aos extratos das plantas inseticidas testadas. $\mathrm{O}$ extrato à base de pó-de-fumo resultou numa eficiência de controle superior a $80 \%$ das larvas $(83,41 \%)$ no segundo dia após a aplicação, sendo que os tratamentos à base de DalNeem e de ramo de cinamomo atingiram esse patamar no quinto e no sexto dias, respectivamente. O tratamento à base de folha de cinamomo não resultou em controle superior a $80 \%$, porém, num valor próximo a esse (76,47\%) (Tabela 1). Em ensaio semelhante, TORRES et al. (2001) observaram que extratos de $\boldsymbol{A}$. indica e de M. azedarach ocasionaram 100\% e 96,7\%

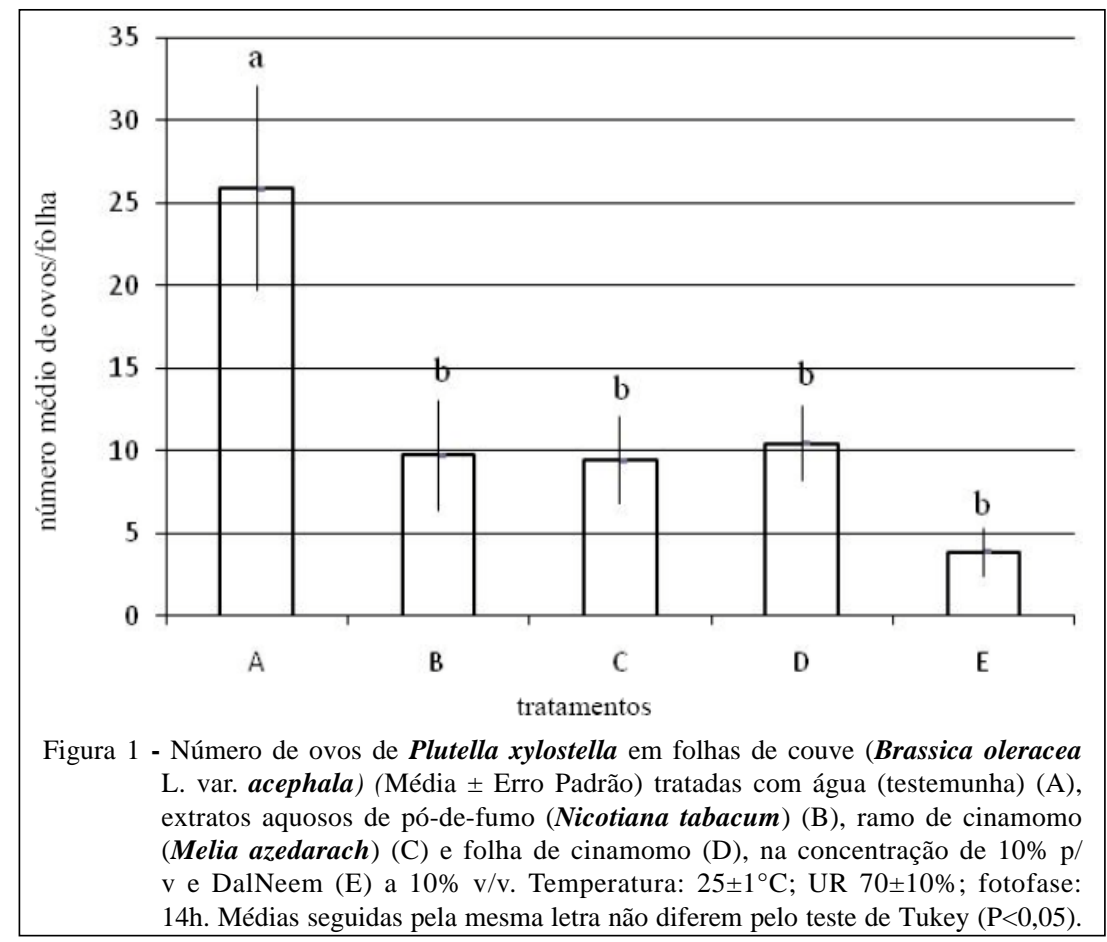

Ciência Rural, v.39, n.2, mar-abr, 2009. 
Tabela 1 - Média acumulada de eficiência de controle (\%), calculada pela fórmula de Abbott, de larvas de Plutella xylostella, alimentadas com folhas de couve (Brassica oleracea var. acephala) tratadas com extratos aquosos de pó-de-fumo (Nicotiana tabacum), ramo de cinamomo (Melia azedarach) e folha de cinamomo, na concentração de $10 \%$ p/v e DalNeem a $10 \%$ v/v. Temperatura: $25 \pm 1^{\circ} \mathrm{C}$; UR $70 \pm 10 \%$; fotofase: $14 \mathrm{~h}$.

\begin{tabular}{|c|c|c|c|c|c|c|c|c|c|c|c|c|c|c|}
\hline \multirow{2}{*}{$\begin{array}{l}\text { Tratamento } \\
\text { Pó-de- } \\
\text { fumo }\end{array}$} & \multirow{2}{*}{$\begin{array}{c}\begin{array}{c}N^{\circ} \text { de } \\
\text { larvas }\end{array} \\
30\end{array}$} & \multicolumn{2}{|c|}{1} & \multicolumn{2}{|c|}{2} & \multicolumn{2}{|c|}{3} & \multicolumn{2}{|c|}{4} & \multicolumn{2}{|c|}{5} & \multicolumn{2}{|c|}{6} & \multirow{2}{*}{$\begin{array}{c}\text { ANOVA: } \\
\text { F }_{(5,15)} ; \mathrm{P} \\
2,71 ; \\
0,0612\end{array}$} \\
\hline & & 69,19 & $\mathrm{~A} \mathrm{a}^{2}$ & 83,41 & A a & 94,67 & $\mathrm{~A}$ a & 94,15 & A a & 100,00 & $\mathrm{~A} \mathrm{a}$ & 100,00 & $\mathrm{~A} \mathrm{a}$ & \\
\hline DalNeem & 33 & 22,96 & $\mathrm{~B}$ ab & 48,15 & $\mathrm{AB}$ a & 65,93 & $\mathrm{~A}$ a & 70,73 & A a & 80,49 & A a & 79,83 & $\mathrm{~A} \mathrm{a}$ & $\begin{array}{c}7,26 ; \\
0,0012\end{array}$ \\
\hline $\begin{array}{l}\text { Ramo de } \\
\text { cinamomo }\end{array}$ & 21 & 16,00 & $\mathrm{~B}$ ab & 43,78 & $\mathrm{AB} \mathrm{a}$ & 43,78 & $\mathrm{AB}$ a & 46,83 & $\mathrm{AB}$ a & 60,00 & $\mathrm{AB}$ a & 82,86 & $\mathrm{~A} \mathrm{a}$ & $\begin{array}{c}2,83 ; \\
0,0540\end{array}$ \\
\hline $\begin{array}{l}\text { Folha de } \\
\text { cinamomo }\end{array}$ & 28 & 6,97 & $\mathrm{Cb}$ & 23,56 & $\mathrm{BC}$ a & 51,41 & $\mathrm{AB}$ a & 55,77 & $\mathrm{AB}$ a & 73,99 & $\mathrm{~A} \mathrm{a}$ & 76,47 & $\mathrm{~A} \mathrm{a}$ & $\begin{array}{l}14,47 \\
0,0000\end{array}$ \\
\hline $\begin{array}{l}\text { ANOVA: } \\
\mathrm{F}_{(3,9)} ; \mathrm{P}\end{array}$ & & \multicolumn{2}{|c|}{4,$64 ; 0,0317$} & \multicolumn{2}{|c|}{2,$40 ; 0,1353$} & \multicolumn{2}{|c|}{2,$47 ; 0,1287$} & \multicolumn{2}{|c|}{1,$57 ; 0,2632$} & \multicolumn{2}{|c|}{1,$25 ; 0,3471$} & \multicolumn{2}{|c|}{ 1,28; 0,3396} & \\
\hline
\end{tabular}

${ }^{1}$ Dias após o tratamento.

${ }^{2}$ Médias seguidas pela mesma letra, maiúscula na linha e minúscula na coluna, não diferem pelo teste de Tukey $(\mathrm{P}<0,05)$.

de mortalidade das larvas da traça-das-crucíferas, respectivamente. Já em ensaio utilizando folhas de $\boldsymbol{N}$. tabacum, BOIÇA JÚNIOR et al. (2005) obtiveram 100\% de mortalidade de larvas de $\boldsymbol{P}$. xylostella no primeiro dia de avaliação e de $89,6 \%$ com $\boldsymbol{A}$. indica após o terceiro dia. LIANG et al. (2003) observaram que todas as larvas de $\boldsymbol{P}$. xylostella alimentadas com folhas de couve tratadas com inseticidas comerciais à base de nim morreram no sétimo dia ou antes.

Nas condições de realização do presente trabalho, foi possível concluir que extratos aquosos a $10 \% \mathrm{p} / \mathrm{v}$ de folha de cinamomo, de ramo de cinamomo e de pó-de-fumo, além de DalNeem a $10 \%$ v/v, reduzem a oviposição de $\boldsymbol{P}$. xylostella em folhas de couve e a utilização de extrato de pó-de-fumo, DalNeem e de ramo de cinamomo controlam eficientemente larvas de $\boldsymbol{P}$. xylostella a partir do segundo, do quinto e do sexto dias após a aplicação, respectivamente.

\section{REFERÊNCIAS}

ABBOTT, W.S. A method of computing the effectiveness of on insecticide. Journal Economic Entomology, v.18, p.265267, 1925.

BOIÇA JÚNIOR, A.L. et al. Efeito de extratos aquosos de plantas no desenvolvimento de Plutella xylostella (L.) (Lepidoptera: Plutellidae) em couve. Arquivos do Instituto Biológico, v.72, n.1, p.45-50, 2005.

CASTELO BRANCO, M.; FRANÇA, F.H. Traça-das-crucíferas, Plutella xylostella (Lepidoptera: Yponomeutidae). In: VILELA, E.F. et al. (Editores). Histórico e impacto das pragas introduzidas no Brasil. Ribeirão Preto: Holos, 2001. p.8589.

CHEN, C. et al. Deterrent effect of the chinaberry extract on oviposition of the diamondback moth, Plutella xylostella (L.) (Lepidoptera: Yponomeutidae). Journal of Applied Entomology, v.120, p.165-169, 1996.
LIANG, G.M. et al. Effects of three neem-based insecticides on diamondback moth (Lepidoptera: Plutellidae). Crop Protection, v.22, n.2, p.333-340, 2003.

LOVATTO, P.B. et al. Efeito de extratos de plantas silvestres da família Solanaceae sobre o controle de Brevicoryne brassicae em couve (Brassica oleraceae var. acephala). Ciência Rural, v.34, p.971-978, 2004.

MACHADO, L.A. et al. Uso de extratos vegetais no controle de pragas em horticultura. Biológico, v.69, n.2, p.103-106, 2007.

MARTINEZ, S.S. Ação do nim sobre os insetos. In: MARTINEZ, S.S. (Ed.). O Nim - Azadirachta indica Natureza, usos múltiplos, produção. Londrina: Instituto Agronômico do Paraná, 2002. p.31-57.

MEDEIROS, C.A.M. et al. Efeito de extratos aquosos de plantas na oviposição da traça-das-crucíferas, em couve. Bragantia, v.64, n.2, p.227-232, 2005.

ROEL, A.R. Utilização de plantas com propriedades inseticidas: uma contribuição para o desenvolvimento rural sustentável. Revista Internacional de Desenvolvimento Local, v.1, n.2, p.43-50, 2001.

TORRES, A.L. et al. Efeito de extratos aquosos de plantas no desenvolvimento de Plutella xylostella (L.) (Lepidoptera: Plutellidae). Neotropical Entomology, v.30, n.1, p.151-156, 2001.

TORRES, A.L. et al. Efeito de extratos aquosos de Azadirachta indica, Melia azedarach e Aspidosperma pyrifolium no desenvolvimento e oviposição de Plutella xylostella. Bragantia, v.65, n.3, p.447-457, 2006.

VENDRAMIM, J.D.; CASTIGLIONI, E. Aleloquímicos, resistência de plantas e plantas inseticidas. In: GUEDES, J.C. et al. (Org.). Bases e técnicas do manejo de insetos. Santa Maria: Pallotti, 2000. 234p. p.113-128.

VIEIRA, P.C.; FERNANDES, J.B. Plantas inseticidas. In: SIMÕES, C.M.O. (Coord.). Farmacognosia: da planta ao medicamento. Porto Alegre/Florianópolis: UFRGS/UFSC, 1999. p.739-754. 